\title{
CMS Pixel commissioning
}

\section{Mauro Emanuele Dinardo* ${ }^{\dagger}$}

University of Colorado

E-mail: Mauro.Dinardo@cern.ch

The CMS pixel detector constitutes the inner core of the tracking system. It is designed to provide three high-precision hits at least per track up to an acceptance in $\eta$ of \pm 2.5 . Together with the ATLAS and ALICE pixel detectors, it represents one of the biggest pixel systems ever built by our community. It consists of about 66 millions pixel cells, 150x $100 \mu \mathrm{m}^{2}$ area, distributed over three concentric barrel layers (48 millions) and four end-cap disks, two on each end of the barrel. I will describe the main features of the system, the project requirements and the performance measured in several tests both on bench and beam. I will then discuss the present status of the detector and the schedule for the installation.

The 16th International Workshop on Vertex detectors

September 23-28, 2007

Lake Placid, NY, USA

*Speaker.

${ }^{\dagger}$ A footnote may follow. 


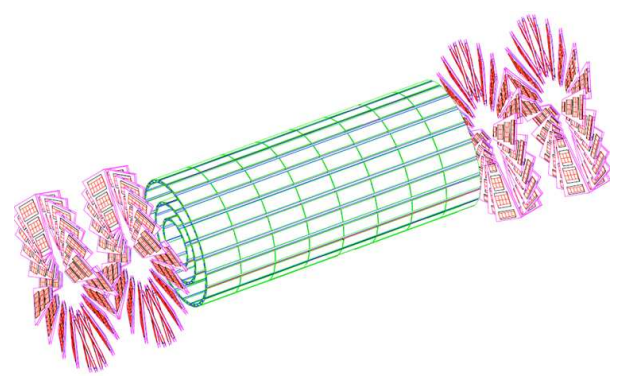

Figure 1: The CMS pixel detector is composed by three barrel layers and four end-cap disks.

\section{Introduction}

The Compact Muon Solenoid (CMS) [1,2] is a general purpose experiment currently being assembled at CERN [3]. The aim of CMS is to study the fundamental properties of elementary particles at the unprecedent energy scale of $14 \mathrm{TeV}$. This will be attained thanks to the new protonproton collider (Large Hadron Collider-LHC). A robust tracking combined with a precise vertex reconstruction within a strong magnetic field of $4 T$ will play a key role to address the full range of physics that would be accessible at this energy. This is realized by implementing an all-Silicon tracking system $[4,5]$.

\section{The Pixel Detector Design}

The pixel detector, see Fig. 1, is composed by three barrel layers (BPIX) at $r$ of 4.3, 7.3 and $10.4 \mathrm{~cm}$ with a total of $\sim 48$ million channels, read out by 11520 ReadOut Chips (ROCs), and four end-cap disks (FPIX) at $z$ of \pm 35.5 and $\pm 46.5 \mathrm{~cm}$ with a total of $\sim 18$ million channels, read out by 4320 ROCs. The pixel cell size of $150 \times 100 \mu \mathrm{m}^{2}$ implies a maximum occupancy of $\sim 0.033 \%$ at full LHC luminosity. The design allows for three pixel hits per track up to $|\eta|$ of $\sim 2.5$; providing:

- seeds for pattern recognition;

- precision vertexing near the Interaction Point (IP);

- fast tracking and vertexing in the High Level Trigger (HLT) using only the pixel information [6].

The ROCs are made in $0.25 \mu \mathrm{m}$ CMOS radiation tolerant technology [7]. The $80 \times 52$ pixel cells of a ROC are organized in double columns with buffers for data and timestamps, 32 and 12 word depth respectively. The readout is analog with zero suppression and the row and column information is encoded on six analog levels as shown in Fig. 2.

The sensors are $n$-on- $n$ type with a p-spray isolation for BPIX and with a partial open p-stop isolation for FPIX [8]. The sensors are connected to the ROCs using In and PbSn bump-bonds for BPIX and FPIX respectively. BPIX and FPIX have good resolution thanks to an average hit cluster size greater than one. BPIX has an average hit cluster size of 2 in $r \phi$ thanks to the large Lorentz angle and a cluster size between 1 and 7 in $z$, depending on the incident angle. FPIX, with 

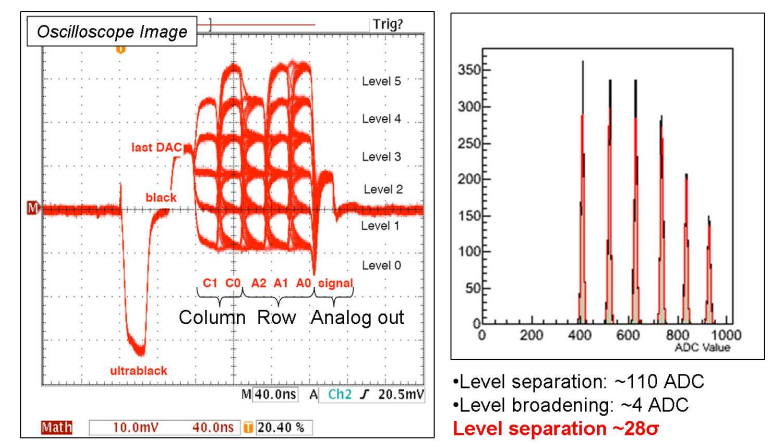

Figure 2: Analog ouput of the pixel front-end electronics. On the left: ROC analog signal seen at the oscilloscope. On the right: histogram of the six levels, used to encode the row and column information, after digitization using a 10 bit ADC.

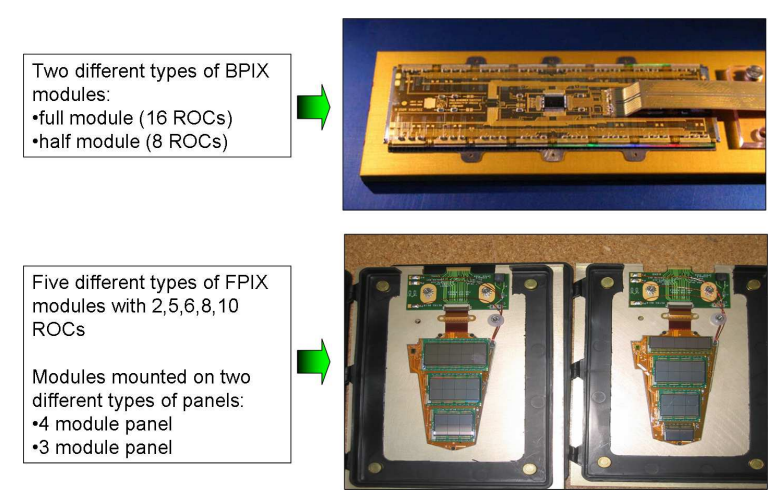

Figure 3: Barrel and end-cap pixel modules.

a turbine geometry with a tilt angle of $20^{\circ}$, presents an average hit cluster size of 2 both along $r$ and $r \phi$, thanks to the Lorentz effect in the first case and to the non-zero incident angle in the latter. The sensor bulk width is $270 \mu \mathrm{m}$, which implies, for a non irradiated sensor, a Landau peak value of $\sim 22000 e^{-}$for a minimum ionizing particle. The sensors have been irradiated up to a proton fluence of $\sim 1.610^{15}$ particles $/ \mathrm{cm}^{2}$, corresponding to about three years of CMS at full LHC luminosity for the innermost layer of the barrel. After irradiation the break-down voltage was still above $600 \mathrm{~V}$, the total collected charge was grater then $70 \%$ and the particle detection efficiency was still greater than $99 \%$.

\section{Pixel Detector Performace}

BPIX is produced, tested and commissioned at the Paul Scherrer Institut (PSI). The modules, see Fig. 3, were produced at a rate of six full modules per day, then thoroughly tested and stored in dry boxes, ready to be mounted on the carbon fiber support structure once the module production was complete.

FPIX is produced and tested in the United States, then commissioned at CERN. FPIX modules were assembled and quickly tested at Purdue University at a production rate of six modules per day. 


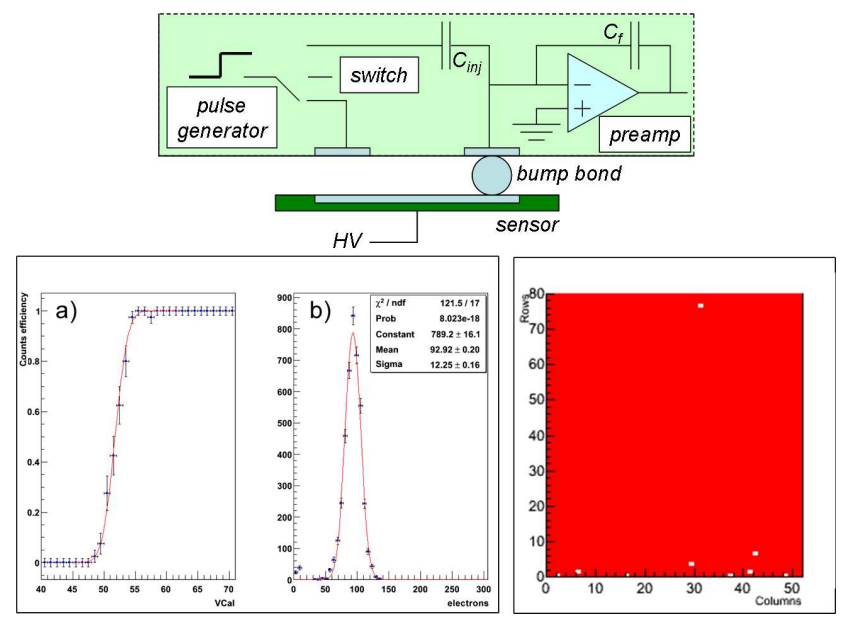

Figure 4: On the top: schematic principle of the ROC internal pulser. On the left: typical threshold curve from one pixel cell together with the noise distribution on a ROC. On the right: map of the bad bump-bonds.

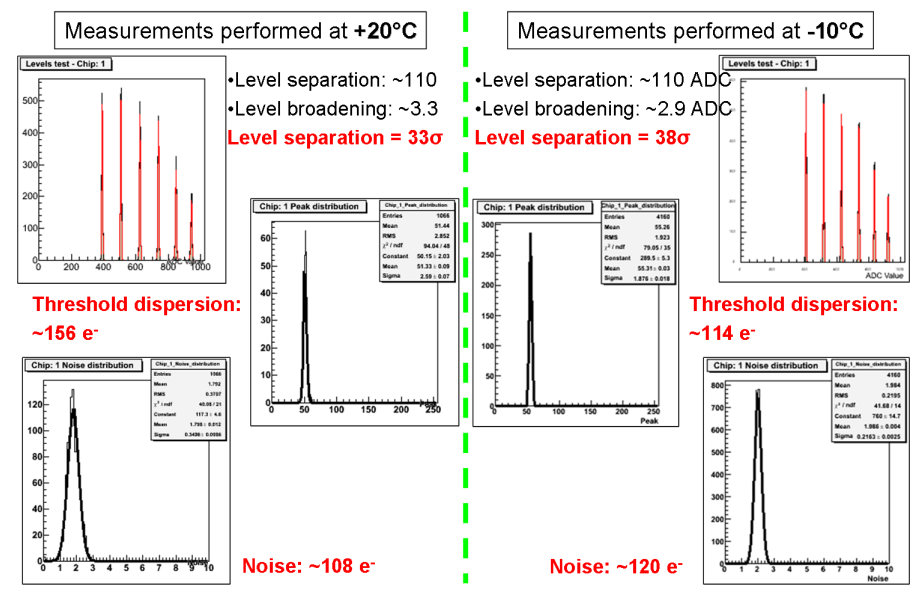

Figure 5: Noise performance of the FPIX prototype pilot detector measured with the final readout system at different temperatures. The $30^{\circ} \mathrm{C}$ variation does not impact the performance.

They were then shipped to the Fermi National Accelerator Laboratory (FNAL). After a visual inspection, modules undergo a two-day thermal cycling process consisting of ten cycles between +20 and $-15^{\circ} \mathrm{C}$. At this point a detailed characterization at $-15^{\circ} \mathrm{C}$ was performed, since the detector will operate at cold temperature to minimize the effects of radiation damage. They were then mounted on the final support structure, quickly tested, and then shipped to CERN where an experiment-like readout system is set up to commission the detector.

All these tests are performed with the ROC internal pulser which emulates the current-pulse that would be generated by a charged particle crossing the sensor. Among several tests, the noise and a map of the bad bump-bonds, are measured as shown in Fig. 4.

A prototype pilot BPIX and FPIX detector, both equivalent to $\sim 4 \%$ of the full system, were also built to pioneer all the assembly and testing procedures. They were built with the same type 

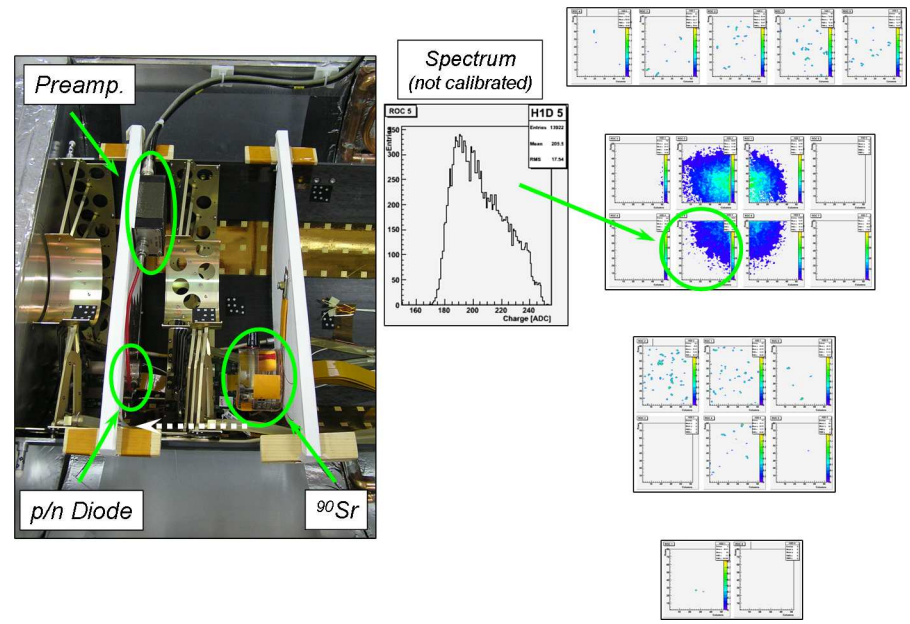

Figure 6: On the left: Strontium-90 beta source set up. On the right: first particles seen by the FPIX detector with the final experiment-like readout configuration.

of mechanical and electrical components as the production detector. The FPIX pilot detector, in particular, was also used for several tests which address the integration into the final experimental set up.

- The pilot FPIX detector was inserted into the full micro-strip tracker. Many tests were performed to learn as much as possible about insertion mechanics, electronics and software. We wanted, for instance, verify that noise was not injected by the strips into the pixel system and vice versa, while the front-end electronics and services were running. Tests were performed both with the pixels grounded to the strip tracker and not. No evidence was found for any performance degradation or interference between pixels and strips;

- A Strontium-90 beta source was used to test the detector and the readout in the experimentlike condition. As shown in Fig. 6 the system was triggered by means of a $p / n$ diode;

- A magnet test was also performed in order to understand the behavior of the FPIX detector in a $4 T$ magnetic field. In particular, we wanted to monitor possible mechanical stress leading to movements due to B-field ramp-up and ramp-down; test possible vibrations of wire-bonds induced by different trigger frequencies. The detector performed as expected and no movement was detected;

- The performance of the pilot FPIX detector was tested at different temperatures and no evidence of change was recorded, as shown in Fig. 5.

\section{Commissioning Strategy}

The strategy we are currently employing for the FPIX detector pre-commissioning consists, first of all, of a sequence of checks: we measure low voltage and high voltage currents; we verify the cleanness of the tip of the optical fibers; we map the sensors for the Detector Control System. Then 
we perform a sequence of tests aimed to compare the detector performance with that measured at FNAL. The tests are performed in two steps:

1. Quick-test aimed to get a fast feedback on the general performance of the detector in order to understand that no accidents occurred during the journey from FNAL to CERN. From the quick-test we can understand if all the ROC wire-bonds are still properly soldered and if all the services are properly working. The test is carried out by measuring on one module per panel the noise and the map of dead cells;

2. Full-test aimed to test the entire detector. For instance we measure threshold and gain-curves, noise and we look for dead channels. We also check that all the high voltage wire-bonds are still properly soldered.

We also run the detector both at room temperature and at $-10^{\circ} \mathrm{C}$. From the experience we are currently gaining during the pre-commissioning in clean room we will define the final commissioning strategy for the installation at P5.

\section{Project Status}

The assembly of the BPIX modules on the support structure will be completed in sixty working days: the first half-shell will be finished by the middle of December and the second one by the end of January. The pre-commissioning of the first $25 \%$ of the FPIX detector has been already done; another $50 \%$ of the detector has been partially pre-commissioned; the pre-commissioning of the remaining 25\% will start soon. We are on schedule for a final installation at P5 in March 2008.

\section{Conclusions}

The construction of the CMS pixel detector is almost complete. We are now performing the pre-commissioning of the detector at PSI for the barrel pixel system and at CERN for the forward pixel system. The initial excellent performance of the modules does not degrade in the final assembled system. Extensive integration tests have beeh carried out to check the compatibility with the final experimental environment, e.g. operation in a $4 T$ magnetic field and inside the micro-trip tracker. We are on schedule for a final installation at P5 in March 2008.

\section{References}

[1] CMS Collaboration, "CMS Physics TDR Volume 1," CERN-LHCC-2006-001 (2006).

[2] CMS Collaboration, “CMS Physics TDR Volume 2,” CERN-LHCC-2006-021 (2006).

[3] CERN web site: www.cern.ch.

[4] CMS Collaboration, "CMS, tracker technical design report" CERN-LHCC-98-06, CMS-TDR-5 (1998).

[5] CMS Collaboration, "Addendum to the CMS tracker TDR," CERN-LHCC-2000-016 (2000).

[6] CMS Collaboration, "The TriDAS Project, Technical Design Report: Data Acquisition and High-Level Trigger," CERN-LHCC-02-26 (2006). 
[7] H.Chr. Kaestli et al., "Design and performance of the CMS pixel detector readout chip" NIM A565 (2006) 188.

[8] G. Bolla, D. Bortoletto, R. Horisberger, R. Kaufmann, T. Rohe, and A. Roya, "Sensor development for the CMS pixel detector," NIM A485 (2002) 89. 